\title{
Aceruloplasminemia with Abnormal Compound Heterozygous Mutations Developed Neurological Dysfunction during Phlebotomy Therapy
}

\author{
Maki Watanabe ${ }^{1}$, Ken Ohyama ${ }^{1}$, Masashi Suzuki ${ }^{1,2}$, Yasunobu Nosaki ${ }^{1}$, Takashi Hara ${ }^{1}$, \\ Katsushige Iwai ${ }^{1}$, Satoshi Kono ${ }^{3}$, Hiroaki Miyajima ${ }^{3}$ and Kenji Mokuno ${ }^{1}$
}

\begin{abstract}
:
Aceruloplasminemia is an autosomal recessive inherited disorder caused by ceruloplasmin gene mutations. The loss of ferroxidase activity of ceruloplasmin due to gene mutations causes a disturbance in cellular iron transport. We herein describe a patient with aceruloplasminemia, who presented with diabetes mellitus that was treated by insulin injections, liver hemosiderosis treated by phlebotomy therapy, and neurological impairment. A genetic analysis of the ceruloplasmin gene revealed novel compound heterozygous mutations of c.1286_1290insTATAC in exon 7 and c.2185delC in exon 12. This abnormal compound heterozygote had typical clinical features similar to those observed in aceruloplasminemia patients with other gene mutations.
\end{abstract}

Key words: aceruloplasminemia, ceruloplasmin gene, anemia, diabetes mellitus, liver hemosiderosis, phlebotomy therapy

(Intern Med 57: 2713-2718, 2018)

(DOI: 10.2169/internalmedicine.9855-17)

\section{Introduction}

Aceruloplasminemia is an autosomal recessive inherited disorder caused by ceruloplasmin gene mutations (1-4). Ceruloplasmin bound with copper has a ferroxidase activity for iron transport. Therefore, the loss of the ferroxidase activity of ceruloplasmin due to gene mutations can cause a disturbance in cellular iron transport. Additionally, it results in iron accumulation in the brain and other organs. The accumulated iron in tissues provides free radicals and lipoperoxidation products which cause oxidative stress, and can eventually lead to tissue and organ damage $(5,6)$.

It is known that the human ceruloplasmin gene has 20 exons, and more than 40 mutations in the ceruloplasmin gene have been identified at many exons. The majority of these mutations can lead to the formation of a stop codon, and the produced protein does not have the necessary structure to perform the enzymatic function in cellular iron transport (5). Aceruloplasminemia is rare disease caused by genetic muta- tions. Most of these genetic mutations are observed within families of a common ancestry (5). In Japan, the frequency of aceruloplasminemia has been estimated to be 1 per $2,000,000$ in the case of nonsanguineous marriages $(6,7)$.

In this report, we describe the clinical features of a patient with aceruloplasminemia having abnormal compound heterozygous mutations in exon 7 and exon 12 of the ceruloplasmin gene. To our knowledge, this combination of mutation sites has not been reported previously.

\section{Case Report}

A 68-year-old woman presented with cognitive decline. She had no relevant family history. She had been treated for several diseases by a primary care doctor and our hospital, before visiting our department (Fig. 1). From about 50 years of age, she had anemia with iron deficiency. Although her anemia had been treated with oral iron supplements, it did not improve. She was diagnosed with diabetes mellitus at 60 years of age. Oral hypoglycemic drugs had been used in the

${ }^{1}$ Department of Neurology, Toyohashi Municipal Hospital, Japan, ${ }^{2}$ Department of Neurology, Nagoya University Graduate School of Medicine, Japan and ${ }^{3}$ First Department of Medicine, Hamamatsu University School of Medicine, Japan

Received: July 20, 2017; Accepted: January 12, 2018; Advance Publication by J-STAGE: April 27, 2018

Correspondence to Dr. Maki Watanabe, watanabe-maki@toyohashi-mh.jp 


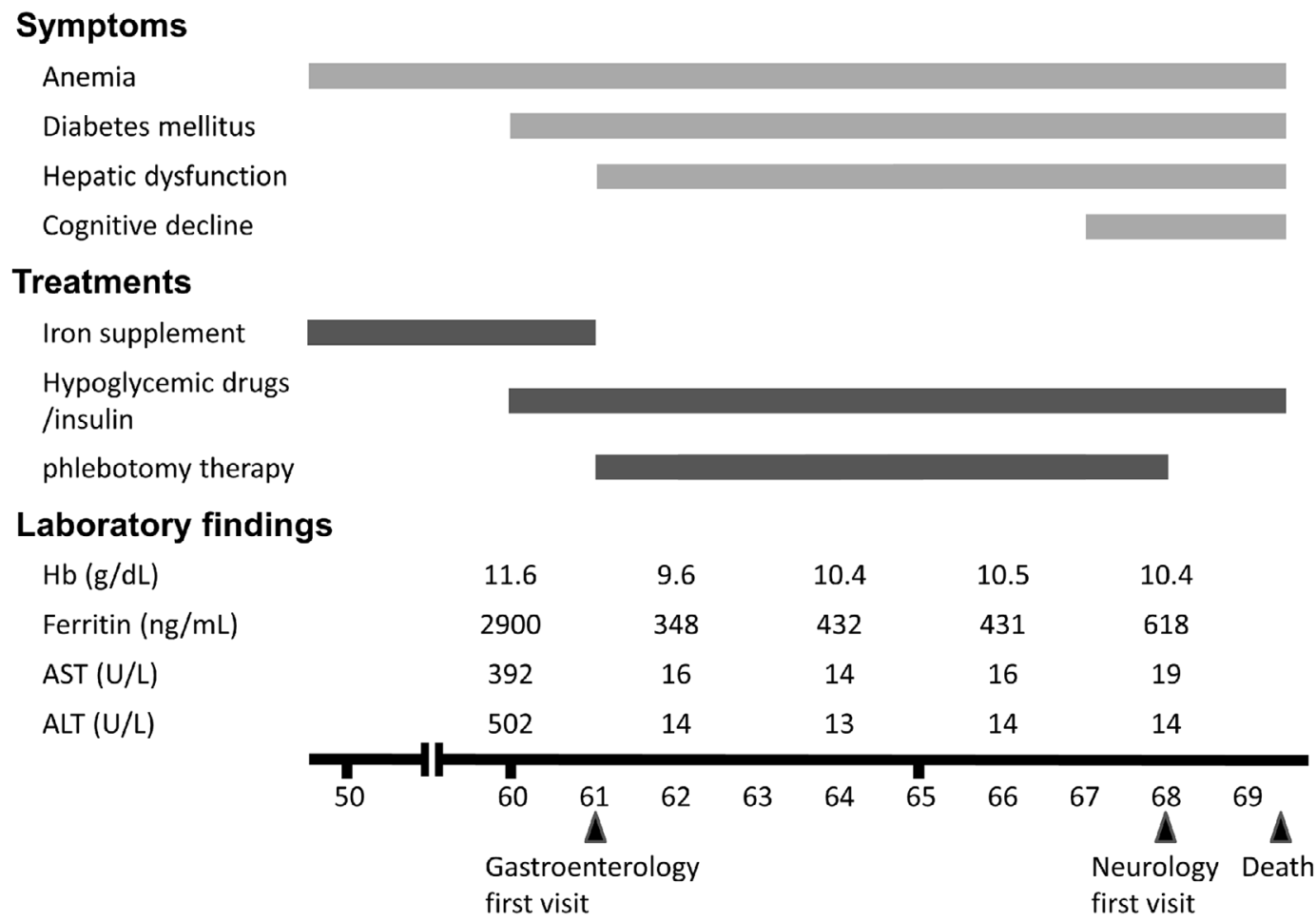

Figure 1. The clinical course of our case is shown. After discontinuing oral iron supplements and initiating phlebotomy therapy, the AST, ALT and ferritin levels improved. However, the cognitive function declined as neurological dysfunction developed during phlebotomy therapy.

initial management of her diabetes mellitus. However, her glycemic control did not improve with these drugs, and treatment with insulin injections was started. She visited an ophthalmologist to check for diabetic retinopathy complications, and no apparent abnormalities were noted. At 61 years of age, she experienced hepatic dysfunction. Her blood chemistry indicated elevated levels of aspartate transaminase (AST) and alanine transaminase (ALT) (AST: 392 IU/L, reference 13-33 IU/L; ALT: 502 IU/L, reference 6-27 IU/L). Therefore, she was referred to the Department of Gastroenterology in our hospital. She was diagnosed with liver hemosiderosis on liver biopsy and was treated with phlebotomy therapy every few months. After discontinuing oral iron supplements and starting the phlebotomy therapy, her anemia was controlled, with hemoglobin levels of approximately $10.0 \mathrm{mg} / \mathrm{dL}$ and the AST and ALT levels decreased to normal. Additionally, her ferritin level improved from $2,900 \mathrm{ng} / \mathrm{mL}$ to $348 \mathrm{ng} / \mathrm{mL}$. On maintaining the phlebotomy therapy, cognitive function decline developed from 67 years of age, and her brain magnetic resonance imaging (MRI) revealed the deposition of iron in the brain parenchyma. Therefore, she visited the Neurology Department in our hospital.

At the first visit, she was $157 \mathrm{~cm}$ tall and weighed 40.2 kg. Her blood pressure was 128/69 (systolic/diastolic) $\mathrm{mmHg}$ in the sitting position, and her resting heart rate was 56 beats per minute (bpm). She was alert and well oriented. Her cognitive function was mildly disturbed at visual memory and word recall. Moreover, her score of the Revised
Hasegawa's Dementia Scale (HDS-R) was 23 points. Although her pupils and range of ocular motion were normal, saccadic eye movement was observed. Other cranial nerve functions and muscle strength of the trunk and extremities were intact. No involuntary movement was observed. The finger-to-nose test and heel-to-knee test identified moderate ataxia in her extremities. She could walk independently, but her gait was short-stepped and wide-based. Additionally, in the one-leg stand test and tandem gait test, she was moderately unstable. Deep tendon reflexes were absent in the extremities. The plantar responses were flexor on both sides. Although her sensations of pain and light touch were intact, her sensation of vibration was moderately decreased in the lower extremities.

The results of blood cell counts and blood chemistry tests are shown in Table. Her blood cell counts indicated anemia with a decreased mean corpuscular volume. The patient's blood chemistry demonstrated decreased levels of serum iron $(\mathrm{Fe})(\mathrm{Fe}: 13 \mu \mathrm{g} / \mathrm{dL}$, reference $45-154 \mu \mathrm{g} / \mathrm{dL})$. Her serum ferritin level was elevated without any oral iron supplements (ferritin: $618 \mathrm{ng} / \mathrm{mL}$, reference 3-120 ng/mL). She had decreased serum copper $(\mathrm{Cu})$ and ceruloplasmin levels $(\mathrm{Cu}: 14.0 \mathrm{ng} / \mathrm{mL}$, reference 66-130 ng/mL; ceruloplasmin: $<0.1 \mu \mathrm{g} / \mathrm{dL}$, reference 21-37 $\mu \mathrm{g} / \mathrm{dL}$ ). Her hemoglobin A1c (HbA1c) was elevated, reflecting diabetes mellitus (HbA1c 6.8\%, reference 4.6-6.2).

Cranial and abdominal MRI was performed. Cranial MRI revealed abnormal low intensity areas in the thalamus, putamen, and cerebellar dentate nucleus in T2-weighted images 
Table. Results of Laboratory Assessments.

\begin{tabular}{lrc}
\hline & & Reference range \\
\hline $\mathrm{WBC}\left(\times 10^{3} / \mu \mathrm{L}\right)$ & 5,930 & $3,040-8,540$ \\
$\mathrm{RBC}\left(\times 10^{4} / \mu \mathrm{L}\right)$ & 447 & $378-499$ \\
$\mathrm{Hb}(\mathrm{g} / \mathrm{dL})$ & 10.4 & $10.8-14.9$ \\
$\mathrm{Ht}(\%)$ & 33.8 & $35.6-45.4$ \\
$\mathrm{MCV}(\mathrm{fL})$ & 75.6 & $85.0-101.0$ \\
$\mathrm{MCH}(\mathrm{pg})$ & 23.3 & $26.8-33.2$ \\
$\mathrm{MCHC}(\%)$ & 30.8 & $30.7-34.0$ \\
$\mathrm{Plt}\left(\times 10^{4} / \mu \mathrm{L}\right)$ & 24.8 & $15.0-36.1$ \\
$\mathrm{AST}(\mathrm{U} / \mathrm{L})$ & 19 & $13-33$ \\
$\mathrm{ALT}(\mathrm{U} / \mathrm{L})$ & 14 & $6-27$ \\
$\mathrm{ALP}(\mathrm{U} / \mathrm{L})$ & 161 & $115-359$ \\
$\gamma-\mathrm{GTP}(\mathrm{U} / \mathrm{L})$ & 13 & $10-47$ \\
$\mathrm{~T}-\mathrm{Bil}(\mathrm{mg} / \mathrm{dL})$ & 0.4 & $0.3-1.2$ \\
$\mathrm{HbA} 1 \mathrm{c}(\%)$ & 6.8 & $4.6-6.2$ \\
$\mathrm{BUN}(\mathrm{mg} / \mathrm{dL})$ & 12 & $8-22$ \\
$\mathrm{Cre}(\mu \mathrm{g} / \mathrm{dL})$ & 0.5 & $0.40-0.70$ \\
$\mathrm{Na}(\mathrm{mEq} / \mathrm{L})$ & 144 & $138-146$ \\
$\mathrm{~K}(\mathrm{mEq} / \mathrm{L})$ & 4.1 & $3.6-4.9$ \\
$\mathrm{Cl}(\mathrm{mEq} / \mathrm{L})$ & 105 & $99-109$ \\
$\mathrm{Fe}(\mu \mathrm{dL})$ & 13 & $45-154$ \\
$\mathrm{UIBC}(\mu \mathrm{g} / \mathrm{dL})$ & 259 & $130-390$ \\
$\mathrm{Ferritin}(\mathrm{ng} / \mathrm{mL})$ & 618 & $3-120$ \\
$\mathrm{Cu}(\mu \mathrm{g} / \mathrm{dL})$ & 14.0 & $66-130$ \\
$\mathrm{Ceruloplasmin}(\mu \mathrm{g} / \mathrm{dL})$ & $<1.0$ & $21-37$ \\
\hline $\mathrm{WBC} \times(\mathrm{m} / \mathrm{b})$ &
\end{tabular}

WBC: white blood cell, RBC: red blood cell, Hb: hemoglobin, Ht: hematocrit, MCV: mean corpuscular volume, $\mathrm{MCH}$ : mean corpuscular hemoglobin, MCHC: mean corpuscular hemoglobin concentration, Plt: platelet, AST: aspartate transaminase, ALT: alanine transaminase, $\gamma$-GTP: $\gamma$-glutamyltranspeptidase, T-Bil: total bilirubin, HbAlc: hemoglobin A1c, BUN: blood urea nitrogen, Cre: creatinine, $\mathrm{Na}$ : serum sodium, $\mathrm{K}$ : serum potassium, $\mathrm{Cl}$ : serum chloride, Fe: serum iron, UIBC: unsaturated iron binding capacity, $\mathrm{Cu}$ : serum copper

(Fig. 2A). Additionally, low intensity areas were observed in the cerebral cortex in $\mathrm{T} 2 *$-weighted images (Fig. 2B). Abdominal MRI showed diffuse low intensity changes in the liver in T2-weighted images (Fig. 2C). A liver tissue biopsy revealed iron deposition in hepatocytes and Kupffer cells. Based on these features, she was diagnosed with liver hemosiderosis.

After obtaining the patient's informed consent, a ceruloplasmin gene analysis was performed at Hamamatsu University School of Medicine. The ceruloplasmin gene analysis revealed two mutations (Fig. 3). A 5-bp insertion in exon 7 (c.1286_1290insTATAC) and a 1-bp deletion in exon 12 (c.2185delC) were observed, and these changes caused frameshift mutations. Therefore, she was diagnosed to have aceruloplasminemia with abnormal compound heterozygous mutations in exon 7 and exon 12.

We discontinued the phlebotomy therapy and planned a therapeutic regimen using the oral iron-chelating drug deferasirox. However, she died while bathing at home, possibly from ischemic heart disease before initiating the iron
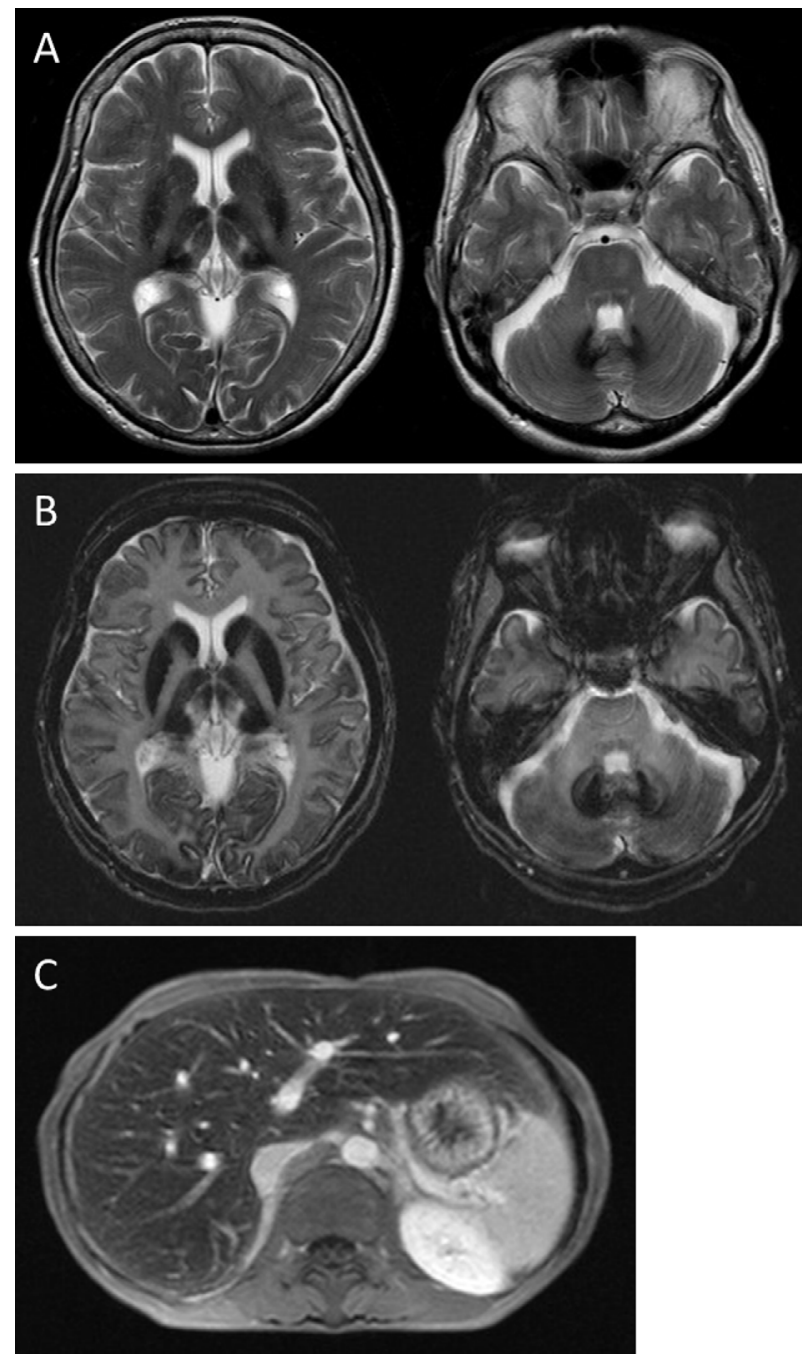

Figure 2. (A, B) T2- and T2*-weighted axial images on brain magnetic resonance imaging (MRI) show low intensity areas in the cerebral cortex, thalamus, caudate nucleus, putamen, and dentate nucleus of the cerebellum. (C) T2-weighted axial images on abdominal MRI show a low intensity area in the liver, thus revealing the deposition of iron.

chelation therapy.

\section{Discussion}

The clinical symptoms of aceruloplasminemia are characterized by anemia, retinal degeneration, diabetes mellitus, and neurological symptoms. The typical course of aceruloplasminemia has been described in previous reports $(5,6)$. In most aceruloplasminemia patients, the symptoms of anemia appear in their twenties or thirties, those of diabetes mellitus appear in their thirties or forties, and neurological symptoms appear in their forties or fifties. In this case, although the onset of these clinical symptoms was delayed, the order of symptoms was similar to that of typical cases, despite the lack of retinal degeneration. Focusing on neurological symptoms, the four important abnormal findings of ataxia, involuntary movement, parkinsonism, and cognitive dysfunction are observed frequently in patients with aceru- 


\section{A}

\section{c.1286_1290insTACAC in Exon7}
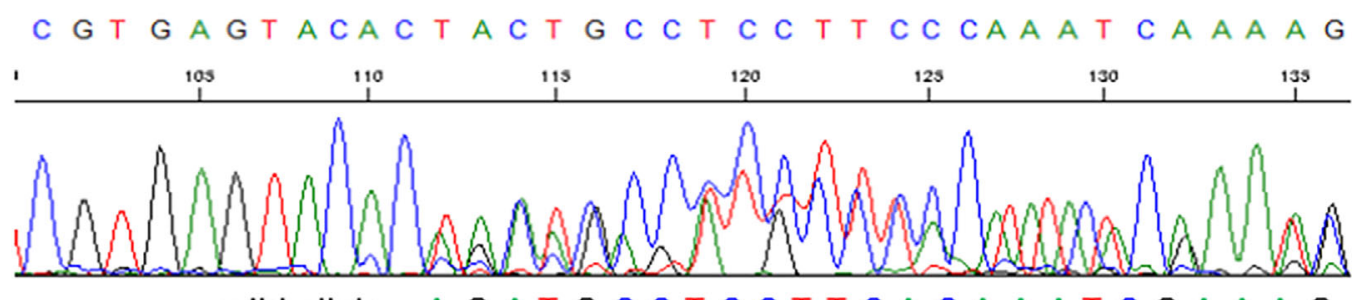

wild-allele A G ATG C C T C C T T C A C A A T C G A A G mutant-allele TA C A C A G A T G C C T C C T T C A C A A A T C

\section{B}

\section{c.2185delC in Exon12}

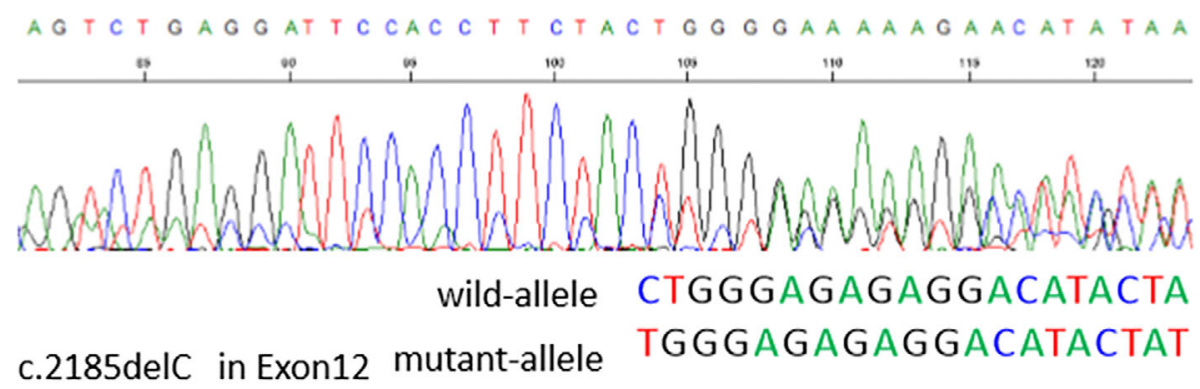

Figure 3. The results of a ceruloplasmin gene analysis. (A) A 5-bp insertion in exon 7 (c.1286_1290insTATAC) and (B) a 1-bp deletion in exon 12 (c.2185delC) are observed. These changes caused frameshift mutations.

loplasminemia $(5,6)$. Although the onset of these neurological symptoms was uncertain in this case, all symptoms except for involuntary movement were clear at her first visit to our department at 68 years of age.

More than 40 mutations of the ceruloplasmin gene have been identified over many exons $(5,6)$. The clinical characteristics are similar despite the differences in the mutation sites, because of the loss of ferroxidase activity of ceruloplasmin. Therefore, there is no genotype-phenotype association in aceruloplasminemia. The clinical features of aceruloplasminemia patients with a homozygous mutation of c.1286_1290insTATAC in exon 7 and with a homozygous mutation of c.2185delC have been reported previously $(2,8)$, and their clinical courses were similar to those of typical aceruloplasminemia patients. In Japan, the ceruloplasmin gene mutations has been described at several other mutation points, such as c.319delT, c.2068delG, c.2482delG, G969S, G873E, W858X, W1017X, 607+1G>A, c. $1209-2 \mathrm{~A}>\mathrm{G}$, and $3019-1 \mathrm{G}>\mathrm{A}(3,9-17)$. However, these mutations were homozygous, and no heterozygous combination has yet been described. This case had abnormal compound heterozygous mutations of c.1286_1290insTATAC in exon 7 and c.2185delC in exon 12, and such a case has not been reported previously. This abnormal compound heterozygote had typical clinical features similar to those in aceruloplasminemia patients with other gene mutations.

In aceruloplasminemia patients, organ disturbances are caused by iron accumulation. To reduce iron accumulation in the organs, iron chelation therapy using deferoxamine and deferasirox, fresh frozen plasma administration, and zinc sulfate therapy have been performed $(5,6,8,18)$. The effectiveness of iron chelation therapy has been described in previous reports $(8,19-21)$. Additionally, some reports have suggested that iron chelation therapy is effective for hepatic iron overload, diabetes mellitus, and neurological symptoms, but not for improving iron accumulation, which can be observed on brain MRI $(15,22,23)$. In our case, the AST and ALT levels were found to be elevated at the time of her first visit to the Department of Gastroenterology, and phlebotomy therapy and the discontinuation of oral iron supplements were carried out to treat her liver hemosiderosis. For these treatments, her AST, ALT, and ferritin levels transiently improved. Previously, her serum AST and ALT levels in the aceruloplasminemia patients were normal $(4,10)$ and slightly elevated $(13,15)$. Therefore, we attributed the elevated transaminases levels to oral iron supplements therapy. Since aceruloplasminemia severely disrupted her iron transport capability, treatment with oral iron supplements might have caused a progression of iron accumulation in the liver, thereby causing liver damage and an elevation of the transaminase levels. We continued the phlebotomy therapy, but the development of neurological symptoms could not be prevented. Although the effectiveness of phlebotomy therapy for aceruloplasminemia patients has not been proven (24-26), we consider that her neurological dysfunction occurred due to disease progression and not by the 
phlebotomy therapy. In contrast, iron chelation therapy has been demonstrated to be appropriate for aceruloplasminemia patients $(8,19-21)$. Iron accumulation in the brain has been observed in patents without any neurological symptoms, and the importance of the early diagnosis of aceruloplasminemia and the early induction of iron chelation therapy has been reported $(5,15)$.

Considering the clinical course of aceruloplasminemia, anemia and diabetes mellitus are the early clinical signs. In aceruloplasminemia patients, anemia appears as microcytic anemia with decreased serum iron and elevated serum ferritin levels (5). In patients with iron-deficiency anemia, the screening of the serum ferritin level is crucial. While irondeficiency anemia results in decreased serum iron and ferritin levels, aceruloplasminemia results in elevated ferritin levels with decreased serum iron levels. In iron overload syndromes, such as aceruloplasminemia, oral iron therapy might therefore accelerate the occurrence of liver damage. Therefore, the screening of the serum ferritin levels is necessary before initiating any therapy for microcytic anemia. Additionally, diabetes mellitus needs insulin for the control of the blood glucose levels, as in insulin-dependent diabetes mellitus $(10,13,27)$. Although the combination of several therapies has been used for the treatment of these symptoms, the effectiveness of these therapies has been shown to be poor, as in the case of phlebotomy therapy for liver hemosiderosis. Therefore, it is important to consider the possibility of aceruloplasminemia when encoutering cases with intractable anemia and diabetes mellitus in order to make an early diagnosis.

In conclusion, we herein described a patient with aceruloplasminemia who demonstrated novel abnormal compound heterozygous mutations in exon 7 and exon 12 of the ceruloplasmin gene. Phlebotomy therapy has only a limited effect with regard to treating liver hemosiderosis in patients with aceruloplasminemia. The early identification of aceruloplasminemia is therefore important in order to timely administer the appropriate therapy and prevent the onset of neurological symptoms.

The authors state that they have no Conflict of Interest (COI).

\section{References}

1. Miyajima H, Nishimura Y, Mizoguchi K, Sakamoto M, Shimizu T, Honda N. Familial apoceruloplasmin deficiency associated with blepharospasm and retinal degeneration. Neurology 37: 761-767, 1987.

2. Harris ZL, Takahashi Y, Miyajima H, Serizawa M, MacGillivray RT, Gitlin JD. Aceruloplasminemia: molecular characterization of this disorder of iron metabolism. Proc Natl Acad Sci U S A 92: 2539-2543, 1995

3. Yoshida K, Furihata K, Takeda S, et al. A mutation in the ceruloplasmin gene is associated with systemic hemosiderosis in humans. Nat Genet 9: 267-272, 1995.

4. Morita H, Ikeda S, Yamamoto K, et al. Hereditary ceruloplasmin deficiency with hemosiderosis: a clinicopathological study of a Japanese family. Ann Neurol 37: 646-656, 1995.
5. Kono S. Aceruloplasminemia: an update. Int Rev Neurobiol 110: 125-151, 2013

6. Miyajima H. Aceruloplasminemia. Neuropathology 35: 83-90, 2015.

7. Miyajima H, Kohno S, Takahashi Y, Yonekawa O, Kanno T. Estimation of the gene frequency of aceruloplasminemia in Japan. Neurology 53: 617-619, 1999.

8. Suzuki Y, Yoshida K, Aburakawa Y, et al. Effectiveness of oral iron chelator treatment with deferasirox in an aceruloplasminemia patient with a novel ceruloplasmin gene mutation. Intern Med 52: 1527-1530, 2013.

9. Yazaki M, Yoshida K, Nakamura A, et al. A novel splicing mutation in the ceruloplasmin gene responsible for hereditary ceruloplasmin deficiency with hemosiderosis. J Neurol Sci 156: 30-34, 1998.

10. Hatanaka Y, Okano T, Oda K, Yamamoto K, Yoshida K. Aceruloplasminemia with juvenile-onset diabetes mellitus caused by exon skipping in the ceruloplasmin gene. Intern Med 42: 599-604, 2003.

11. Miyajima H, Takahashi $\mathrm{Y}$, Kono S, Hishida A, Ishikawa K, Sakamoto M. Frontal lobe dysfunction associated with glucose hypometabolism in aceruloplasminemia. J Neurol 252: 996-997, 2005.

12. Hida A, Kowa H, Iwata A, Tanaka M, Kwak S, Tsuji S. Aceruloplasminemia in a Japanese woman with a novel mutation of CP gene: clinical presentations and analysis of genetic and molecular pathogenesis. J Neurol Sci 298: 136-139, 2010.

13. Ogimoto M, Anzai K, Takenoshita H, et al. Criteria for early identification of aceruloplasminemia. Intern Med 50: 1415-1418, 2011.

14. Fujita K, Osaki Y, Harada M, et al. Brain and liver iron accumulation in aceruloplasminemia. Neurology 81: 2145-2146, 2013.

15. Tai M, Matsuhashi N, Ichii O, et al. Case of presymptomatic aceruloplasminemia treated with deferasirox. Hepatol Res 44: 12531258, 2014.

16. Nagata S, Ikegaya N, Ogino $\mathrm{S}$, et al. The resection of thyroid cancer was associated with the resolution of hyporesponsiveness to an erythropoiesis-stimulating agent in a hemodialysis patient with aceruloplasminemia. Intern Med 56: 805-810, 2017.

17. Kohno S, Miyajima H, Takahashi Y, Inoue Y. Aceruloplasminemia with a novel mutation associated with parkinsonism. Neurogenetics 2: 237-238, 2000.

18. Kuhn J, Bewermeyer H, Miyajima H, Takahashi $\mathrm{Y}$, Kuhn KF, Hoogenraad TU. Treatment of symptomatic heterozygous aceruloplasminemia with oral zinc sulphate. Brain Dev 29: 450-453, 2007.

19. Miyajima H, Takahashi Y, Kamata T, Shimizu H, Sakai N, Gitlin JD. Use of desferrioxamine in the treatment of aceruloplasminemia. Ann Neurol 41: 404-407, 1997.

20. Skidmore FM, Drago V, Foster P, Schmalfuss IM, Heilman KM, Streiff RR. Aceruloplasminaemia with progressive atrophy without brain iron overload: treatment with oral chelation. J Neurol Neurosurg Psychiatry 79: 467-470, 2008.

21. Bove F, Fasano A. Iron chelation therapy to prevent the manifestations of aceruloplasminemia. Neurology 85: 1085-1086, 2015.

22. Finkenstedt A, Wolf E, Hofner E, et al. Hepatic but not brain iron is rapidly chelated by deferasirox in aceruloplasminemia due to a novel gene mutation. J Hepatol 53: 1101-1107, 2010.

23. Roberti Mdo R, Borges Filho HM, Goncalves CH, Lima FL. Aceruloplasminemia: a rare disease - diagnosis and treatment of two cases. Rev Bras Hematol Hemoter 33: 389-392, 2011.

24. Brissot P, Troadec MB, Bardou-Jacquet E, et al. Current approach to hemochromatosis. Blood Rev 22: 195-210, 2008.

25. Bethlehem C, van Harten B, Hoogendoorn M. Central nervous system involvement in a rare genetic iron overload disorder. Neth J Med 68: 316-318, 2010.

26. Lozano-Varela M, Carrera-Alonso E, Plaza-Palacios G. Aceru- 
loplasminemia: an entity to consider in patients with anemia. Rev Esp Enferm Dig 106: 360-361, 2014.

27. Vroegindeweij LH, van der Beek EH, Boon AJ, et al. Aceruloplasminemia presents as Type 1 diabetes in non-obese adults: a detailed case series. Diabet Med 32: 993-1000, 2015.
The Internal Medicine is an Open Access article distributed under the Creative Commons Attribution-NonCommercial-NoDerivatives 4.0 International License. To view the details of this license, please visit (https://creativecommons.org/licenses/ by-nc-nd/4.0/).

(C) 2018 The Japanese Society of Internal Medicine Intern Med 57: 2713-2718, 2018 